\title{
Enquête
}

Archives de la revue Enquête

6 | 1998

La description I

\section{La bonne description}

Good Description

\section{Fernando Gil}

\section{(2) OpenEdition}

\section{Journals}

Édition électronique

URL : http://journals.openedition.org/enquete/1493

DOI : 10.4000/enquete. 1493

ISSN : 1953-809X

\section{Éditeur :}

Cercom, Éditions Parenthèses

\section{Édition imprimée}

Date de publication : 1 octobre 1998

Pagination : 129-152

\section{Référence électronique}

Fernando Gil, «La bonne description », Enquête [En ligne], 6 | 1998, mis en ligne le 15 juillet 2013, consulté le 19 avril 2019. URL : http://journals.openedition.org/enquete/1493 ; DOI : 10.4000/ enquete. 1493

Ce document a été généré automatiquement le 19 avril 2019. 


\title{
La bonne description
}

\author{
Good Description
}

\section{Fernando Gil}

« Le concret, c'est ce qui est intéressant. La description. D’objets, de paysages, de personnages ou d'actions. En dehors, c'est du n'importe quoi. »

Claude Simon, Le Monde, 19 septembre 1997.

1 D'après le Petit Robert, décrire c'est « représenter dans son ensemble, soit par écrit soit oralement ». Pour le Littré, également, représenter et dépeindre sont les significations principales. Décrire comme représenter désignent des actes de la pensée. Mais décrire signifie bien d'autres choses si on se réfère à des acceptions plus anciennes qui subsistent dans la philosophie de la description. Décrire et description tiennent de l'abstrait et du concret, de l'actif et du passif. Décrire signifie représenter, dépeindre, retracer, exhiber, au XVIII écrire, consigner, formuler (Grimm): la description tient de l'abstrait. Mais décrire signifie aussi présenter - darstellen (Grimm) -, énumérer les caractères de quelque chose, détailler : la description tient du concret ou d'un mouvement vers un concret. La description colle à la chose décrite qui est son contenu. Le latin inclut sous descriptio la descriptio locorum de la peinture ainsi que la délimitation, la détermination, la fixation (des charges d'un magistrat, par exemple). La description évoque alors la figure se détachant $\mathrm{du}$ fond et le bord qui trace une frontière. Elle démarque l'objet qu'elle veut saisir dans sa concrétude, son modèle est la perception. La description est «l'ornement du discours qui consiste à peindre sous les couleurs les plus vives » (Littré).

2 La description ressortit aussi à la " réceptivité » et à la "spontanéité ». Elle copie la chose passivement. Tel est d'ailleurs son sens en tant que représentation, elle reproduit le donné. Mais dans la description la chose se pose elle-même ; du côté du sujet, on retrouve cette dimension d'activité dans délimitation, fixation, « remplissement » - v. vollschreiben, par exemple implere paginam (Grimm) -, qui sont d'autres significations de décrire. La forme achevée de cette description créatrice s'inspire de la géométrie et de la phoronomie. Décrire une courbe c'est la construire: les astres décrivent leurs mouvements en les effectuant. 
3 La description est donc copie et construction, représentation indéterminée autant que présentation en "chair et en os ». Elle est une démarche paradoxale. Ces hésitations lexicales sous-tendent et nourrissent la philosophie et l'épistémologie de la description sous forme de problèmes et d'apories. Dire que la description oscille entre l'abstrait et le concret conduit à chercher des catégories qui lui soient propres. Y a-t-il des concepts descriptifs, à mi-chemin entre les protocoles d'observation et les concepts explicatifs ? Dire que la description est réceptive et active à la fois c'est s'interroger sur son objectivité. La description « fidèle » se veut factuelle. Mais étant une « construction » elle contient des a priori, des interprétations. Comment concilier l'exigence d'objectivité avec l'arbitraire de l'interprétation? On appellera la première question catégorisation de la description, la seconde statut de l'observation. L'évaluation de la bonne description en dépend. Qu'est-ce qui est bon dans la bonne description, d'où la description reçoit-elle sa valeur? De sa teneur informative - et cette teneur se jauge-t-elle à l'aune de la « fidélité »? Ou de ses virtualités opératoires, dans un contexte d'enquête - mais alors à quoi l'« opérativité » se mesure-t-elle?

4 Concrétude et factualité pointent vers les contenus, abstraction de la représentation et interprétation du donné sont l'œuvre du sujet. Par le biais des catégories et par le biais de l'observation la description engage et le sujet et l'objet. La catégorisation se rapporte éminemment à la matière à décrire, l'observation à l'intention de décrire. La frontière entre description et interprétation, suivant la terminologie consacrée, est intraçable. Toute description est "chargée ", sinon de théorie, tout au moins d'attentes qui en prédéterminent l'orientation. Et les contours mouvants de la description paraissent interdire d'emblée toute catégorisation. Néanmoins, nous savons que fixer un fait est autre chose qu'interpréter et qu'entre le perçu et l'explication l'observation a sa place.

5 Nous partirons de l'épistémologie de la description; les descriptions linguistiques sont en effet la forme élémentaire et la cellule germinale de toute description. Nous ferons ensuite référence à la philosophie de la description; nous terminerons enfin par une application de nos résultats au cas paradigmatique examiné par Gilbert Ryle et Clifford Geertz : que décrit un certain mouvement des yeux?

6 Les langues véhiculent des théories du monde dont les verbes, les noms, les adjectifs, les adverbes, les prépositions sont les descripteurs naturellement adaptés. La langue est le modèle princeps d'un ajustement des concepts et des objets qui joue directement dans l'épistémologie de la description.

7 Qu'est-ce qu'une bonne description linguistique ? La grammaire de Ronald Langacker se propose de mettre en évidence le fait que le langage est par nature cognitif et que la cognition linguistique s'accompagne d'une référence multiple au sujet. Le choix d'une description (que Langacker appelle «image») recouvre un nombre considérable de décisions, et les choix retenus et les rejets correspondants s'étayent de principes descriptifs bien déterminés, même s'ils ne sont pas conscients ${ }^{1}$. Dans l'exemple qu'analyse Langacker, Floyd a cassé un verre, le schéma sujet-verbe-complément d'objet représente une situation très simple, «atomique " : l'action d'un homme a changé une chose, en lui ôtant le vinculum (pour le dire comme Leibniz) qui lui apportait son unité « substantielle». 
8 La phrase aurait pu être énoncée d'une façon appropriée en des contextes fort différents, qui rendraient la description linguistique plus ou moins intéressante. Par exemple, le verre est cassé par inadvertance dans un magasin regorgeant de bibelots et il est alors peu probable qu'on en arrive à une enquête. Mais on peut supposer qu'au cours d'une réception Floyd a pris un marteau qu'il a ensuite lancé contre un verre à boire. De quelles décisions interprétatives la phrase Floyd a cassé un verre est-elle l'aboutissement?

Il faut en premier lieu que le locuteur choisisse les genres d'entités qui seront les constituants de l'événement, en d'autres termes il doit définir l'ontologie de sa description (Langacker dit « base »). À « personne », « marteau », « verres » et « casser », trois entités et une action perçues par le locuteur, il aurait pu parler d'impulsions nerveuses, de contractions musculaires, des lois de la balistique ou de la propagation des discontinuités dans une substance cristalline. Il aurait pu aussi sélectionner des entités abstraites, telles que " coup de marteau ", "force ", " action ", " puissance », « intégrité structurale " - ou encore des parties et non des touts, par exemple " côtés ", «éclats ", "fragments", "bras", "tête du marteau». On aurait eu alors des phrases très différentes de Floyd a cassé un verre.

Commentaire. Le locuteur a choisi l'ontologie naturelle du nom et du verbe et l'évidence perceptive, au lieu (1) des ontologies non immédiatement observables de la physique et de la biologie ou (2) d'une ontologie à dominante verbale et adjectivale désignant des processus et propriétés qui ne sont pas tous directement perçus, ou encore (3) d'une ontologie "méréologique». Son choix serait vraisemblablement celui de tout native speaker d'une langue indo-européenne (d'autres langues, admettons-le, préféreront les processus aux entités). En témoignerait la tonalité forcément bizarre des phrases formulées dans les ontologies (1) (2) et (3), y compris pour le physicien des solides et le neurologiste, ou pour le disciple de Lesnievski ${ }^{2}$. Une saillance cognitive se trouve naturellement attachée aux touts, aux entités matérielles, aux actions et à la perception directe, ce qui rend ces ontologies sémantiquement non acceptables.

Cela étant, le locuteur dispose encore de plusieurs possibilités de description qui forment des chaînes d'actions distinctes. Langacker en imagine trois, ce n'est pas limitatif. À la place de Floyd a cassé un verre, le locuteur aurait pu dire, sans bizarrerie, Floyd a cassé un verre avec un marteau (peut-être l'option la plus naturelle). Dans la première proposition le locuteur n'a pas mentionné le marteau parce qu'il le considère comme un prolongement du bras, et la description fait intervenir deux participants : $(F-M) \Rightarrow V$ (où $F=$ Floyd, $M$ $=$ marteau, et $\mathrm{V}=$ verre). La deuxième proposition décompose la chaîne en deux actions avec trois participants : $F \Rightarrow M=>V$, plus exactement $(F \Rightarrow M) \&(M \Rightarrow V)$. Troisième choix possible, également recevable, Floyd a propulsé son bras, et le marteau a fait voler un verre en éclats. Le locuteur considère ici une partie du corps de Floyd comme un objet qu'il lance. La phrase modélisera d'ailleurs deux représentations différentes, suivant que la partie du corps est seulement le bras : $(F \Rightarrow B \Rightarrow M \Rightarrow V)$, ou qu'elle inclut le marteau : $(F \Rightarrow>(B-M)=>V)$

Commentaire. Ces descriptions relèvent d'orientations cognitives différentes. La première rapporte simplement un événement dans l'espace-temps (faute de renseignements, on admet qu'il n'y a pas un contexte privilégiant Floyd ou le verre, par exemple une question au sujet de l'un ou de l'autre), la seconde s'intéresse autant à l'événement qu'à l'instrument qui l'a provoqué (la " causalité efficiente »), la troisième met en avant le processus qui a abouti à l'effet et l'effet lui-même, c'est-à-dire la causalité "finale » de l'événement. Floyd a cassé un verre décrit une action comme si elle était un fait, le geste est raconté au passé et dans le perfectif aspectuel : il est objectivé sans qu'on tienne compte de son agencement. À l'inverse, 
Floyd a propulsé son bras, etc. saisit l'action presque comme si elle se déroulait encore, la description attire l'attention sur sa dynamique interne, "en acte ". Le fait y est décrit comme une action. Entre ces deux extrêmes, la description d'un fait et la description d'un acte, la proposition Floyd a cassé un verre avec un marteau se laisse comprendre soit comme rabattant l'action sur le fait, en l'objectivant, soit, à l'opposé, comme suspendant le verre cassé au geste et au marteau.

D'autres facteurs de sélection ont joué, tel le «niveau de spécificité », du vague ( " marteau ») au précis (on aurait pu avoir « le marteau à panne fendue avec un manche en bois»). Telle aussi la "visée » (scope) de la description, c'est-à-dire, ce qui est sélectionné de l'ensemble de la situation (une réception) pour en faire l'objet de la prédication. Le locuteur s'est seulement intéressé à la chaîne des actions : l'origine et la transmission de l'énergie, de F à V. Il a ignoré la couleur des yeux de Floyd, son effort pour attraper le marteau, les éclats de verre qui ont volé dans toutes les directions et ont peut-être atteint quelqu'un. Il aurait pourtant pu prétendre exprimer tout cela, par la phrase Avec les yeux bleus en feu, Floyd a pris le marteau et a cassé un verre en mille morceaux, en blessant plusieurs personnes avec ses éclats qui sont allés partout. Il aurait pu encore, on ajoutera, se restreindre à un seul tronçon de la chaîne des actions et se borner à déclarer : Floyd a pris le marteau et l'a lancé.

12 À la visée de la description appartient aussi son "profil », c'est-à-dire ce à quoi le locuteur attribue une position dominante, et ce qu'il choisit comme « figure » et comme « fond ». Ainsi, il profilera la description à partir de la chaîne des actions (Floyd a cassé un verre), ou de l'interaction entre le marteau et le verre (le marteau a cassé un verre), ou du mode d'affection, la « résistance » du verre (un verre s'est cassé facilement). Enfin, les voix se trouvent dans une relation figure-fond. Fond dans la voix active, le verre est figure dans la passive (un verre a été cassé par Floyd).

Commentaire. Profil et figure-fond renvoient directement à un point de vue, c'est-àdire à l'observation (ils jouent aussi dans la catégorisation). Les descriptions se font d'après ce qu'on veut regarder: ce que fait Floyd ou ce qui arrive au verre, ou au verre par rapport au marteau ou par rapport à Floyd.

Visée et niveau de spécificité conditionnent la catégorisation de la description. Les exemples de Langacker suggèrent que la catégorialité descriptive se place entre le trop et le trop peu d'information. Floyd a cassé un verre est une description middlerange, elle n'a pris en compte ni un maximum (cf. Avec les yeux bleus en feu, Floyd a pris le marteau, etc.), ni un minimum des possibilités descriptives (cf. par exemple Floyd a pris un marteau).

Il en a été de même dans les choix précédents. L'ontologie du nom (personnes et choses) et du verbe (actions) est plus concrète que les ontologies scientifiques «invisibles", qu'il s'agisse de la physique cristalline et de la neurologie ou de dynamiques cachées. En revanche, cette ontologie est plus abstraite que ne le serait la seule description de parties (cf. «bras»), à la place des touts qu'elles composent (par exemple " homme ", a fortiori « Floyd »). De même, les descriptions Floyd a cassé un verre, Floyd a propulsé son bras, etc. et Floyd a cassé un verre avec un marteau sont à un niveau intermédiaire. Elles esquissent des situations-type, dans le système de la langue et dans l'image du monde qu'elle projette : le fait, l'action, leur conjonction moins stable. Les trois descriptions se trouvent à égale distance de, disons, Floyd... (suit sa biographie, son état civil, ses projets d'avenir, ses lieux de vacances habituels, ses vins préférés, à la limite sa "notion complète ») a cassé un verre (fait dans l'usine $\mathrm{X}$, en..., etc.) - ainsi que de, disons, quelqu'un a cassé quelque chose. On aurait du mal à appeler description aussi bien la première que la deuxième formulation. 
13 Quelles conclusions convient-il de tirer de ces analyses? Elles semblent à première vue contradictoires. En premier lieu, face à une matière à décrire, une description (toute description) est choisie parmi une multitude de descriptions possibles. Apparemment banale, cette constatation a des conséquences fortes; elle ne se limite pas à entériner un conventionnalisme de bon aloi. Sans compter les phrases «bizarres» (sémantiquement mal formées) que l'ontologie de la langue conduit à écarter, la description Floyd a cassé un verre a été sélectionnée parmi une douzaine d'alternatives. Mais cet éventail ne désigne pas des possibilités descriptives d'un même fait qui se situeraient au même rang : chaque description démarque un fait différent. Aussi s'impose immédiatement cette conséquence que la qualité de la description ne consiste pas dans sa conformation avec le donné. Le donné est ce que chaque description décide de prélever d'une matière « informe » en se donnant pour tâche de le décrire, il lui est conforme eo ipso, sans possibilité de réfutation. Dans une terminologie carnapienne, les descriptions sont trivialement «internes» au cadre de référence qui est le leur, elles s'épuisent dans l'intention dont elles se soutiennent. La description est par définition adéquate car elle crée son propre objet. De même, le niveau de spécificité, le profil ou la visée, la figure et le fond de la description s'accordent nécessairement avec l'intention du descripteur, c'est lui qui décide de l'objet et de la finesse de l'attention, de ce qu'il paraît pertinent ou non de prendre en considération. À aucun de ces titres une description n'est intrinsèquement meilleure qu'une autre. Le problème serait alors de savoir s'il existe une description qui ne soit pas bonne.

Ensuite, il ressortirait de l'analyse de Langacker que la description choisie est compatible avec les descriptions rejetées, qui sont également compatibles entre elles. Tel serait aussi le cas des ontologies sous-jacentes aux descriptions. Certes, les entités de la physique ne sont pas celles du «langage des choses» (Carnap), mais seule une métaphysique moniste prohibe leur coexistence. Toujours dans le même esprit, l'ontologie chosale des langues indo-européennes est compatible avec l'ontologie fluante du hopi puisqu'elles constituent des cadres de référence différents. Le monisme du «langage " occulte la diversité éventuellement non commensurable, partant compatible, des langues. Pour sa part, l'expérience du monde, chosale et fluante à la fois, ne tranche pas en faveur d'une ontologie ou de l'autre.

On pourra penser que cette compatibilité extérieure des univers du discours corrige la relativité interne des descriptions. Si, dans le même univers (a fortiori en des univers différents), les faits dépeints par les descriptions sont compatibles, on est tenté d'imaginer un univers leibnizien d'expressions partielles d'une même réalité. C'est sur cet univers que misent les théories " cumulativistes $^{3}$ " de la vérité scientifique. Mais on oublie là que la partialité de la description est une donnée structurelle de l'observation, elle appartient à sa condition. Aussi chacune de nos descriptions linguistiques n'est compatible avec les autres qu'en théorie. Aussitôt énoncée, elle occupe tout «l'univers du discours » et il n'y a qu'un seul univers du discours à la fois qui occupe tout l'espace de l'enquête. Le remplacement d'une description par une autre déloge son univers propre et déclenche une autre enquête, la compatibilité additive des descriptions est l'envers mythologique du positivisme.

En effet, l'acquis définitif - éminemment métaphysique - de l'histoire et de la sociologie des sciences est d'avoir mis au jour dans la démarche scientifique ce que Kant et Fichte 
ont dénommé finitude. C'est ce que "partiel» signifie. Toute enquête cognitive a un horizon, cela fait que l'horizon se déplace avec le mouvement de la recherche mais aussi que l'enquête à chaque moment est contenue dans ses limites. L'ensemble que forment les descriptions et la théorie se trouve contraint en permanence par une clôture interne qui joue dans le sens description $=>$ théorie autant que dans le sens théorie $\Rightarrow>$ description. Dire qu'un ensemble de descriptions constitue l'assise d'une théorie revient à expliquer qu'à l'instar des descriptions linguistiques au regard de l'expérience, la théorie choisit un certain nombre de descriptions dont elle fait sa "base ", de préférence à d'autres qui définiraient une autre base. Ces descriptions délimitent la portée empirique de la théorie. En même temps, la théorie fixe le domaine des « bonnes questions » - c'est l'autre nom de l'intention descriptive -, qui mèneront à des descriptions nouvelles. En cela consiste la coadaptation des observations et des théories. Elle est évolutive, autrement dit elle s'affine peu à peu, la visée des descriptions se précisant d'autant (la « science normale " peut élargir cette visée).

17 Des théories différentes se référeront au même corpus de descriptions, la description n'implique pas une interprétation unique - mais l'idée d'un ensemble convergent de descriptions différentes, s'entr'exprimant pour décrire la notion complète de la réalité, est un rêve de visionnaire, même à titre d'idée régulatrice. La finitude de la connaissance scientifique réside en ce qu'elle s'élabore dans le cadre d'une enquête, celle-ci et non pas une autre, pour un certain point de vue (la théorie et le chercheur qui la repense) et dans les bornes de son corpus de descriptions. Chaque théorie est un habitus intériorisé par le chercheur - ce n'est là quelque chose ni de contingent, ni de moderne - et aussi le système de coordonnées de ses questions et de ses descriptions. L'esprit humain n'est pas ainsi fait qu'il puisse embrasser toutes les questions possibles et décrire le monde sous tous les points de vue. La compatibilité des descriptions dans une certaine niche de l'expérience n'autorise pas à extrapoler jusqu'à une vue "externe» unique sur la connaissance : celle-ci constituerait un point de vue infini, une contradictio in adjecto. On n'est pas à même d'imaginer la Grande Description qui concilierait les descriptions où prendraient appui toutes les théories d'une discipline. Et le rêve de la Grande Théorie qui réconcilierait les théories à la racine de «toutes » les intentions descriptives ne tient pas compte de la difficulté encore plus grande à rendre les théories compatibles. Elles se fabriquent pour et avec un certain nombre de descriptions. Mais, à la différence de ces dernières, mouvantes et singulières, toujours en voie de céder la place à de nouvelles représentations des faits, les concepts sont rigides et les théories systématiques.

Compatibilité reste un concept négatif, agnostique, alors que pour assurer la cumulativité des descriptions il serait besoin de principes capables d'unifier les ontologies et les descriptions. C'est ce qui se produit lorsque théories et observations en viennent à se coadapter, comme cela a été le cas dans la mécanique quantique. L'opposition ondecorpuscule s'est relativisée, d'abord par la description mathématique, dans la même équation (en fait deux équations), de l'énergie et de la quantité de mouvement des " corpuscules" en termes "ondulatoires", ensuite par la relativisation des concepts mêmes de corpuscule et d'onde dans la théorie quantique des champs. Tant que les comportements quantiques sont tout simplement ondulatoires sous certaines conditions et corpusculaires sous d'autres, les descriptions réfèrent des faits différents. 

descriptions sont $a$ priori adéquates car elles posent ce qu'elles décrivent. Le cadre décide des bonnes questions, que le chercheur "entraîné » transforme en descriptions (chaque observation, chaque expérience). Le dilemme entre la fidélité au fait et l'arbitraire de l'interprétation - c'est-à-dire, le problème du statut de l'observation - trouve ici sa solution de principe, qui ne peut pas être générale. "L'interprétation", ce sont les paramètres de pertinence des descriptions, l'horizon d'attente qui les entoure - et la «factualité » se trouve assurée au départ. L'interprétation signifie cette prédétermination du regard, elle vient avant et non après, sans déformer le donné car il n'y pas de donné cognitif avant la description qui l'instaure. (Cette constatation métaphysiquement neutre est compatible avec le réalisme et avec l'idéalisme).

On comprend que, comme l'a remarqué Wittgenstein, des exécutions différentes d'une œuvre musicale puissent être également justes. Si la partition fixait à l'avance le sens unique de l'œuvre, une seule interprétation serait alors autorisée. Mais les exécutions reposent sur des descriptions différentes de la partition, qui sont " compatibles " parce qu'elles décrivent des réalités différentes. Car l'œuvre est et n'est pas la même, suivant le «niveau de jeu» qui dans l'exécution musicale représente l'équivalent des échelles scientifiques de l'observation. La partition reste identique pour tous ses lecteurs (on la reconnaitra donc toujours comme telle). Ce premier niveau constitue la description univoque d'un seul état de choses. La lecture de la partition est obligée, suivant une méthode de description exclusive de toute autre, savoir le déchiffrement de la partition selon un code. Mais l'œuvre n'est pas la même aux autres niveaux de jeu. Tempi, rubato, toucher, valeurs, etc. différents, jusqu'à la possibilité de l'invention (cf. cadences), instituent des descriptions différentes. Comme les descriptions scientifiques, elles se font d'après la « théorie » de l'interprète - son point de vue sur l'œuvre - et se trouvent eo ipso avérées. Mais elles signifient autre chose que la seule conception du sens de la partition, l'« interprétation » s'appuie sur une certaine distribution de ses éléments (de même que l'interprétation d'un tableau s'étaye d'une configuration, qu'on privilégie, de son dessin, ses couleurs, ses masses). Toutefois, ici aussi on ne saurait rêver d'une Grande Interprétation réunissant toutes les exécutions «justes ». Les œuvres se font pour des destinataires, des individus - il s'agit d'une détermination d'essence -, qui ne sont pas prêts à entériner n'importe quelle exécution, qui apparaitra juste à d'autres.

Mais il ressort aussi des variations sur Floyd que le regard s'oriente selon des vecteurs dont l'origine doit être cherchée dans la matière à décrire. Si l'observation se greffe sur les problèmes d'une "théorie » - il en va ainsi déjà dans la théorisation permanente à laquelle l'expérience quotidienne nous oblige -, la catégorisation se laisse guider par l'objet. Les choix du locuteur de Floyd a cassé un verre ne se font pas au hasard et ils ne se bornent pas à corroborer l'intention de rapporter ce qui s'est passé dans la réception, pour la bonne raison qu'ils ne se connaissent pas eux-mêmes (c'est en quoi les descriptions linguistiques sont instructives). Le locuteur a choisi une ontologie chosale et verbale, une certaine présentation de l'occurrence (les actions $F \Rightarrow M=>V$, rassemblées en un événement singulier), la voix active, une certaine saillance - cet événement-là - qui se détache de la toile de fond de la réception, une teneur informative « moyenne ». Grosso modo, on peut supposer que d'autres participants à la réunion auront prêté la même 
attention aux mêmes faits, et effectué le même choix de mettre l'accent sur la chaîne complète des actions et de considérer le marteau comme un appendice du bras. Sans son effet final, l'événement n'est pas suffisamment intéressant, et la distinction entre bras et marteau (Floyd a propulsé son bras, et le marteau, etc.) n'est en rien pertinente. En revanche, le geste de Floyd tel que la description l'a consigné est une conduite pour le moins étrange.

Pour donner un exemple d'un autre type - l'exemple et le type sont les catégories descriptives par excellence -, une description du psychisme qui ne prendrait pas en compte les "gaffes" en société se priverait de quelque chose qui invite à enquêter : un comportement involontaire et embarrassant est intrinsèquement intéressant, quoi qu'il en soit de son interprétation. D'abord, la gaffe détonne, fait "figure ", au regard des règles de la convivialité. Ensuite, notre horizon théorique incite à demander si cet involontaire ne relève pas de l'inconscient, étant donné que le même horizon commande de comprendre les phénomènes appelés inconscients (ou de les déconstruire moyennant leur réinterprétation). C'est en ce sens que l'involontaire et l'embarrassant constituent matière à décrire, sans que pourtant nos attentes théoriques prévoient le comportement déterminé qu'est la gaffe. Aussi, un comportement à la fois involontaire et embarrassant s'impose de soi-même à l'attention avant qu'il ne soit interprété, par exemple comme un acte manqué, parfois au sens propre : il n'est pas exclu que Floyd ait déplacé sur le verre son envie de casser la tête de quelqu'un. De simplement linguistique, cette description deviendrait alors psychologique.

Il y a du plus et du moins intéressant dans la matière à décrire. Cet intérêt valide la description de l'intérieur, en la convertissant en réalité observée, digne d'être pensée et narrée. Elle est la réalité cognitive tout court ; en dehors de la description, c'est du n'importe quoi informe. La validation s'achèvera par la confirmation intersubjective de la description: mutatis mutandis comme un bon roman, qui finit toujours par trouver son public.

\section{III}

La philosophie de la description tourne autour de cette validation interne même lorsqu'elle la nomme autrement. La description est pour Husserl spécifique d'un certain type de savoir, celui des «sciences descriptives». Il l'étudie avec la «méthode de la clarification", dans les Ideen I, en rapport avec l'exemple, le type et la variation. La doctrine husserlienne de la description est au service d'une certaine philosophie de la connaissance, la "phénoménologie comme théorie descriptive de l'essence des purs vécus ", suivant le titre du $\$ 75$ des Ideen I. Il est d'autant plus frappant que l'on retrouve l'exemple, le type ou la variation chez des penseurs aussi différents que Kant, Goethe, Mach ou Wittgenstein. Par-delà les usages particuliers, ces catégories font système et leur portée rejaillit sur la description en général. La bonne description tient de l'exemple, du type ou de l'expérience imaginaire, elle s'auto-valide pour autant qu'elle est " exemplaire ».

Chez Husserl, la bonne description suppose la clarté et celle-ci s'évalue par la proximité. La clarté parfaite consiste dans la "proximité absolue ${ }^{4}$ ». La clarification combine deux démarches : rendre clair (intuitif) ce qui ne l'est pas, accroître ou intensifier (steigern, le verbe de Goethe) ce qui l'est déjàs. Cette opération est déjà descriptive, la clarification aboutit à un remplissement, elle élimine les «éléments représentés à vide». La 
clarification porte sur un donné confus où les "essences " se trouvent "entrelacées " (mitverflocht). Pour le fixer, on prendra appui sur des singularités exemplaires ${ }^{6}$, la détermination se poursuivant à l'aide de variations imaginaires des exemples (§ 70). On désignera comme essence ce qui demeure invariant au cours des variations. Husserl développe ensuite l'opposition entre sciences descriptives et sciences exactes dont la géométrie est le modèle. Les sciences descriptives sont « concrètes » (\$ 72), « anexactes par essence et non par hasard », elles s'agencent par des « concepts morphologiques portant sur des types vagues de formes qui seraient directement saisis en se fondant sur l'intuition sensible et qui seraient, quant aux concepts et à la terminologie, fixés de façon aussi vague que le sont eux-mêmes ces types ${ }^{7} »$. Toutefois, étant typiques les essences descriptives sont aussi " génériques » et, dans cette mesure, rigoureuses. Ainsi, même si les contenus de la perception, du souvenir ou du vouloir sont fluants et vagues, la méthode de l'exemple et des variations permet de dégager leurs essences génériques avec rigueur8.

C'est ce qui transparait de la " démarche progressive » de la clarification: «Même si les intuitions individuelles [...] sont déjà suffisamment claires [...], il subsiste un défaut de clarté en ce qui concerne les déterminations plus précises des essences entrelacées; il faut donc qu'on serre de plus près les singularités exemplaires ou qu'on en fournisse de nouvelles qui soient mieux adaptées et où les traits singuliers appréhendés dans la confusion et l'obscurité pourraient prendre du relief et accéder alors au rang des données les plus claires ${ }^{9}$. " Husserl ne s'attarde pas sur les critères du choix des exemples, ils posent pourtant un problème de fond. L'essence invariante se détermine moyennant des variations sur des exemples, et pour ce faire il faut que ceux-ci se révèlent être «bien adaptés ». Mais selon quels critères ? L'intention descriptive n'y suffit pas, les singularités doivent exhiber leur propre "adaptation » (sous peine de circularité, l'essence ne peut pas orienter le choix des exemples).

L'adaptation de l'exemple - sa vocation à faire paraitre l'essence, comme pour le morceau de cire de Descartes ${ }^{10}$ - présuppose certes la coadaptation des théories et des descriptions, chaque singularité nouvelle se profile contre une histoire, dans le cadre d'une enquête. Mais la description intéressante est la singularité " exemplaire", où les traits singuliers "prennent du relief», comme le dit Husserl, non celle qui se limite à confirmer les descriptions antérieures. Kant en a proposé une théorisation complexe qui repose précisément sur l'opposition de ces deux régimes ; elle s'exprime par la distinction entre Exempel et Beispiel ${ }^{11}$. L'Exempel exemplifie seulement, il est l'illustration d'une règle générale dont il représente simplement un " cas particulier ». À l'inverse, le Beispiel n'est pas «pris » parmi d'autres exemples de statut identique, il est « introduit» (v. anführen). Il dépasse l'exemplification pour atteindre à l'exemplarité, son but est la " compréhension ». On construit et on compare le Beispiel à d'autres exemples possibles dans un système de variations -, il ne spécifie pas une règle mais invente un modèle. Le Beispiel est problématique, il y aura de "bons» et de moins bons exemples, mais il possède une force (Kraft) que Kant ne reconnaît pas à l'Exempel.

La note du $\$ 52$ de la «Doctrine de la Vertu» explique que l'exemple (Beispiel) fait fusionner le «particulier» avec l'« universel» et l'«abstrait», l'intelligible, avec le " concret», le sensible, comme si les deux relations ne faisaient qu'une seule: «Un exemple n'est que le particulier [das Besondere] (concretum) représenté comme compris sous l'universel d'après des concepts (abstractum) et ce n'est que la présentation [Darstellung] théorique d'un concept.» 
Chez Kant, la relation particulier-universel se rattache au contenu significatif de la description, la relation abstrait-concret à sa référence : l'exemple condense en soi les deux acceptions de l'identité. Par une sorte de surcompensation en présence, il efface l'incertitude de la description et l'accroche à un donné12. En cela réside la force de l'exemple. Kant s'en servira dans les contextes les plus divers, entre autres dans la "Méthodologie de la Raison Pure Pratique », au travers de l'expérience imaginaire de l'enfant qui reconnaît la «pure vertu » dans l'histoire d'un homme qui a su résister aux pressions les plus fortes. Frédéric est l'exemple brillant (glänzendes) des Lumières, l'enseignement et la conduite du Christ sont l'exemple et la preuve d'une conduite absolument pure, la Révolution Française restera à tout jamais le «signe historique » d'une cause de progrès inhérente à l'homme, dont il est l'auteur ${ }^{13}$. L'exemplarité du génie se trouve au cœur de la Critique de la faculté de juger.

Dans l'Introduction à l'« Analytique des Principes », Kant touche au choix du bon exemple indirectement, en rapport avec l'exercice de la faculté de juger ${ }^{14}$. La "seule et grande utilité » de l'exemple consiste à aiguiser la faculté de juger ; il se résumerait à la fonction « d'aide » mentionnée dans la première préface de la Critique (A XVIII-XIX). Cependant, cet aiguisement est le seul guide de la faculté de juger car celle-ci n'obéit pas à des règles, son mode d'être est la pratique. Chercher une règle pour la faculté de juger « en général » entraîne une régression à l'infini : " elle ne peut pas être saisie mais seulement exercée » (B 172) et dans ces termes le bon exercice de la faculté de juger se trouve suspendu à l'exemple. Celui-ci n'en est pas moins la condition que le produit, sa portée constitutive s'avérant d'autant. On parvient au bon exemple en comparant des possibilités, en cherchant des analogies, en inventant des situations imaginaires. Mais la force de l'exemple se trouve renfermée dans l'exemple même, elle est l'autre nom de l'évidence de l'homme probe ou de la révolution (le Fils de Dieu a lui aussi provoqué une « révolution dans le genre humain $\left.{ }^{15} »\right)$.

1 En d'autres termes, c'est l'objet qui s'ex-pose. On se tournera maintenant vers le Husserl d' Expérience et jugement. La matière à décrire se présente sur le mode d'«excitations » intensives, insistantes, qui font pression sur la conscience ${ }^{16}$. On dirait aujourd'hui que cette matière constitue un paysage d'attracteurs et de saillances, au-delà de la factualité brute et en deçà de l'interprétation théorique, l' intéressant" est sa modalité. "L'intérêt pris à l'objet de l'expérience» est la première strate de l'enquête ${ }^{17}$. Wittgenstein dira que l'esthétique est descriptive parce qu'elle attire l'attention ${ }^{18}$.

L'exemple doit être en quelque sorte typique, il tend naturellement à devenir paradigmatique (ce que la langue atteste dans sa traduction du terme grec). Les bons exemples de Kant sont des prototypes, au sens de George Lakoff: on ne donnera pas l'autruche pour exemple d'un oiseau, les membres d'une même classe logique n'ont pas tous le même statut sémantique ${ }^{19}$. On peut supposer que Husserl a emprunté à Goethe le thème du type, en relation étroite avec le principe de la variation. Le type est un modèle de concordances ou de ressemblances et de différences, une norme, une image générale, un parangon de comparaisons, un modèle du tout ${ }^{20}$. Il est le concept-clé de l'anatomie comparée, les comparaisons conduisant à des «monographies" de deux sortes. Si l'on " décrit » les espèces animales d'après le type, « on n'a plus besoin de comparer l'animal à l'animal, il suffit de confronter les descriptions et la comparaison se fait d'elle-même ». La 
deuxième sorte de monographie consistera à décrire complètement (durchbeschreiben) une certaine partie anatomique à travers les principaux genres zoologiques, une «comparaison instructive (belehrende)» s'obtenant de la sorte ${ }^{21}$. Dans les deux cas, l'intelligibilité des descriptions est ostensive. Concept descriptif et non explicatif, le type ne charrie pas une théorie (il n'est pas theory loaden). Typique et non imitative, la description dessine les reliefs de l'expérience, qu'elle signifie ${ }^{22}$.

L'expérience imaginaire ou "de pensée » constitue un mélange d'exemple et de type elle est un " exemple exemplaire "-, on y envisage certaines circonstances dont découle l'anticipation d'une certaine conséquence. Les circonstances sont conçues par une "méthode de la variation ${ }^{23}$ ». Pierre Duhem a dénoncé dans l'expérience imaginaire une pétition de principe, elle se présente " comme si elle était réelle et pose comme fait ce qu'elle a présupposée ${ }^{24}$ ». Cette critique oublie que dans une «bonne» expérience de pensée, les circonstances imaginées esquissent des situations d'extrémalité l'extrémalité est une forme de typicité - autrement dit, des constructions qui reposent sur un minimum de présupposés, censés être des présupposés inquestionnables. Les inférences faites sur eux conduiront alors à des conséquences également indubitables, la réduction du champ de l'expérience se double dans la même mesure de l'éclat de la conclusion imparable. Le réel se laisse simuler par l'imaginaire parce que l'ensemble imaginé témoigne d'une évidence qui lui vient de son dépouillement - c'est ce que l'expérience imaginaire ajoute à l'exemple, au type et à la variation qui sont ses ingrédients.

Depuis les bateaux de Galilée, les expériences imaginaires appartiennent à l'attirail ordinaire de la physique. Dans les sciences sociales, elles ont une place de choix dans toute recherche prenant pour objet les origines qui sont en soi des situations d'extrémalité. Ainsi le xVIII ${ }^{e}$ siècle, amoureux des origines, abonde en expériences imaginaires, depuis le problème de Molyneux à l'enfant de Kant, à la Galatée de Rousseau, à la statue de Condillac: Victor de l'Aveyron, situation d'extrémalité en acte, condense les attentes à la racine de ces expériences. Plus près de nous, le « voile d'ignorance » de John Rawls est un modèle parfait de cette extrémalité placée aux origines dont on anticipe un effet. La déduction des deux principes de la justice se fait d'elle-même si les contractants ne connaissent ni leur propre position sociale, économique et politique, ni celles des autres, ni encore leur force ou intelligence propres - et s'ils connaissent quelques « faits généraux de la société ", relatifs à l'existence en société, à l'économique et au politique, ainsi que les lois de la psychologie humaine ${ }^{25}$.

Comme Peter Strawson le note à l'endroit de sa propre philosophie, la «métaphysique descriptive » constitue « une enquête qui ne demande pas à être justifiée ${ }^{26}$ ».

\section{IV}

La catégorialité médiane de la description, un pied dans l'abstrait l'autre dans l'empirique, réside donc dans sa vocation d'exemplarité et de typicité, qui en fait le référentiel virtuel pour d'autres descriptions. Comme le type, la bonne description est le germe d'un système de variations, Floyd a cassé un verre en appelle à d'autres découpages de la matière à décrire. "Notre investigation ne porte pas sur les phénomènes, mais, comme on pourrait dire, sur les possibilités des phénomènes", ce principe de Wittgenstein ne vaut pas moins dans la recherche scientifique qu'en philosophie ${ }^{27}$. Le 
type n'est pas une copie, sans pour autant s'étayer d'une théorie. De même, la description n'invente pas le donné qu'elle établit par le geste même d'observer et qui est par ailleurs toute la réalité à laquelle elle peut accéder. Le réel reste irrémédiablement voilé, pour reprendre l'expression de Bernard d'Espagnat, et l'interprétation s'est constituée par avance, elle est l'hypothèse à la source des «bonnes questions» qui vont orienter les rayons attentionnels, comme le dirait Husserl.

Une ostension sur fond de relativité, telle sera la formule de la bonne description. Tendanciellement évidente en termes de catégorialité, la description est relative aux modalités et aux échelles d'observation et ne peut pas outrepasser sa finitude intrinsèque. Il n'est pas de Grande Description, «ce que nous nommons "description", ce sont des instruments destinés à des applications particulières (besondere) ${ }^{28} »$. Le statut d'être de la description est transitoire, fugace. Elle fixe un fait pour le convertir immédiatement en une interrogation que les hypothèses et les théories prendront en charge et qui suggère d'autres hypothèses et ouvre vers de nouvelles descriptions. Sa fonction est opératoire, la mauvaise description est la description insuffisamment informative pour susciter un questionnement (Floyd a regardé un verre, Le marteau était à sa place, La marquise est sortie à cinq heures) ou celle dont l'excès d'information étouffe toute "prise d'intérêt » dans l'œuf: Avec les yeux bleus en feu, Floyd a pris un marteau à panne fendue avec le manche en bois, puis il a propulsé son bras et le marteau a cassé un verre en mille morceaux, en blessant plusieurs personnes avec ses éclats qui sont allés partout, dont Betty, Paul et Fred qui étaient pourtant à la fenêtre et regardaient en bas. La précision maniaque décourage le questionnement, on peut supposer qu'il porterait sur l'auteur de la phrase, non sur la matière qu'elle décrit.

Essayons pour finir d'appliquer nos résultats à la description linguistique suivante : Floyd a baissé et relevé la paupière de son ceil gauche très vite ${ }^{29}$. Comment faut-il désigner le fait décrit, est-ce un tic, un clin d'œil, la parodie du clin d'œil d'un tiers, l'imitation d'un geste $\mathrm{vu}$ sur scène? Ces interrogations correspondent à des attentes descriptives qui catégorisent le mouvement de l'œil à l'intérieur d'un éventail de comportements « exemplaires », « prototypiques » (des types idéaux). L'expérience sociale commune en constitue le référentiel théorique. Un autre plan de questions, plus complexe, amplifie immédiatement ce premier ordonnancement. Son cadre reste l'expérience ordinaire telle que le langage naturel la décrit, mais la catégorisation est maintenant abstraite, elle désigne des propriétés exprimées par des expressions adjectivales : spontané, volontaire, etc. - non des comportements individués par des descriptions indéfinies : un tic, un clin d'œil, une parodie, une imitation. Il en va de même des points de vue, qui dressent une stratification de l'expérience. Ils se placent aux embranchements d'un graphe arborescent.

En fait, les comportements-type sont bien plus de quatre et ils se démultiplient en une foule d'autres catégorisations, au regard de plusieurs points de vue possibles. Ceux-ci s'énoncent sous la forme de questions qui conduiront à l'enquête scientifique, à un troisième niveau ${ }^{30}$. La description s'achève lorsqu'on dispose d'une information satisfaisant les attentes d'identification du plissement de l'œil, d'après les points de vue et aux niveaux d'observation où l'on se place. L'esquisse qui suit ignore les marges de compénétration du donné, c'est-à-dire sa "surdétermination », et les chevauchements 
des disciplines qui en découlent ${ }^{31}$. Il y aurait d'autres arrangements possibles tout à fait appropriés.

(I). Le mouvement est-il spontané ou volontaire? S'il est spontané, constitue-t-il un mouvement réflexe, un automatisme, un dérèglement nerveux, une « somatisation » ? La description linguistique tic recouvre ces diverses possibilités qui intéresseront des disciplines scientifiques distinctes. Dans le cadre des sciences humaines, seule l'hypothèse de la somatisation se révèle être intéressante, la psychologie étant la principale science concernée (et la description est finie de son point de vue). Il en va de même pour la physiologie ou pour la neuro-physiologie, si le mouvement d'œil est classé comme un réflexe ou un autre automatisme. Dans tous les cas la description se prolongera par des descriptions plus fines, suivant les échelles d'observation propres aux disciplines (contextes expérimentaux, etc.).

41 Si le mouvement est volontaire, il n'intéresse pas la neurophysiologie mais il pourra intéresser la psychologie et il intéressera les «disciplines de l'intersubjectivité ». Sa bonne description sera désormais clin d'œeil, mais cette désignation n'est qu'une tête de chapitre. Plusieurs catégorisations différentes sont ici possibles. En tenant compte des situations envisagées par Ryle, on se demandera peut-être à ce moment si Floyd a fait le clin d'œil en son nom propre ou s'il a imité sans malice le clin d'œil d'un tiers. On rencontrera plus loin la citation malicieuse; la distinction sincère-insincère aurait pu intervenir à ce niveau de description, on suivrait alors un autre cheminement. En cela réside la liberté de choix des cadres de référence.

(II). Ce deuxième embranchement distingue le discours direct, « en première intention ", de la citation, en "seconde intention ». Ce clin d'oil est-il un geste propre ou la reproduction $d u$ clin d'œil d'un tiers? S'il s'agit d'une citation, disons d'un acteur - pour en rester au typique -, il n'y aura pas d'enquête intersubjective. On simplifie bien sûr, il serait loisible de se demander pourquoi, dans un certain contexte intersubjectif, on fait la citation.

Celle-ci sera intéressante pour l'esthétique théâtrale si d'une façon ou d'une autre elle se rapporte au jeu de scène, par exemple si Floyd veut être acteur. La description (achevée) conduira à poser une question sur la qualité de l'exécution, qui est une question sui generis: elle ne fait pas problème (n'est pas pertinente) dans les autres descriptions considérées.

(III). En admettant que le mouvement de paupière se fasse en première intention, le pas suivant pourrait consister à savoir si le clin d'œil s'adresse à soi ou à autrui. Floyd exprime peut-être devant un miroir son amour-propre ou, moins narcissiquement, son amour de soi - ou alors il se moque de lui-même, plus ou moins amicalement. Ces attitudes intéresseraient de façon fort différente la psychanalyse et l'analytique existentielle de Binswanger pour qui la névrose représente la perte d'une estime «bien ordonnée » de soi. Quoi qu'il en soit, l'identification du comportement s'arrête là pour la psychologie. Mais il est probable que Floyd a cligné de l'œil à l'intention d'un vis-à-vis et on pourra se demander alors si son clin d'œil est amical ou inamical. Dans un cas comme dans l'autre, la discipline de référence est dorénavant la « psychologie sociale » et le clin d'œil sera pris dans sa fonction la plus fortement signifiante. Que veut-il dire? Chaque description sera une réponse à cette question et convoquera immédiatement des hypothèses d'interprétation.

(IV). Le clin d'œil amical témoigne d'une connivence ou de la recherche d'une connivence, il intéresse la sociologie de l'empathie, la philosophie du visage et du regard... 
L'interrogation sur le sens s'approfondit et se complique. Le clin d'œil amical comprend toute une gamme de possibilités, sans oublier les formes du « neutre », le tiers non exclu ni amical ni inamical - (V) le clin d'œil instrument de la "fonction phatique », ou ayant une fonction déictique: "à ton tour!», ou simple signe de reconnaissance. Plusieurs embranchements sont donc possibles, que le comportement soit amical ou neutre. Si on choisit de ne pas les prendre, l'identification du clin d'œil se trouve terminée dans les deux hypothèses.

(VI). La signification inamicale est particulièrement intéressante, car elle va à l'encontre du codage social. Puisque le mouvement de paupière de Floyd déforme la signification typique du clin d'œil (amicale ou neutre), seule une recherche contextuelle l'identifiera comme inamical, qu'il se réfère à la relation de Floyd avec son vis-à-vis, ou qu'il rapporte le clin d'œil d'un tiers (le contexte y étant alors la relation avec le vis-à-vis, l'interprétation ne fera pas problème). Telles seraient les deux branches du questionnement. Si la complicité du clin d'œil est feinte, la description désigne un comportement hypocrite (mais l'inamical peut cacher une demande d'amitié), si le mouvement de paupière reproduit inamicalement le clin d'œil du tiers, il sera typiquement une parodie qui le tourne en dérision. On retrouve là la citation, mais la deuxième intention ne se superpose pas et ne gomme pas ici le discours direct, elle est mise au service d'une première intention malicieuse.

À chaque niveau d'observation, d'après un certain point de vue, les descriptions sont en principe incompatibles. Le mouvement de paupière est spontané ou volontaire, il se fait en nom propre ou il est une citation, il s'adresse à soi ou à autrui, il est amical, neutre ou inamical, il vise intentionnellement l'interlocuteur ou évoque un tiers. D'autres cartes de possibilités descriptives sont pensables où les descriptions s'excluent aussi les unes les autres. Chacune des alternatives obéit et actualise une intention descriptive. En termes de liberté, le plissement de l'œil est-il volontaire ? En termes de sociabilité, le clin d'œil est-il amical ? En termes d'authenticité, est-il sincère, hypocrite, parodique?

Chaque description est une réponse à l'une de ces questions qui définit son horizon. Elle enclenche une enquête, descriptive à un second niveau, suivant de nouveaux paramètres - par exemple, les réquisits de la communication lorsqu'on s'interroge sur la fonction phatique du clin d'œil -, interprétative ensuite. Pour quelle raison Floyd se montre-t-il amical, inamical, ricaneur, content de lui ?

Si les descriptions sont incompatibles dans le cadre d'un point de vue, les points de vue tendent à organiser l'expérience suivant une stratification. Comme Kant le remarque dans l'Appendice de la "Dialectique Transcendantale », la pensée du genre et de l'espèce forme le contenu même de l'entendement. L'esprit humain est ainsi fait qu'il hiérarchise l'expérience.

L'enquête est inachevable, les descriptions sont ses points d'arrêt provisoires, elles fixent un fait qui apparaît au regard comme ostensiblement intéressant, suivant une certaine orientation théorique. Le fait est interprété, c'est-à-dire expliqué, par une ou plusieurs théories en compétition. Soit l'explication ne conduit pas à un second regard sur la description, soit elle le fait, à la suite d'une "résistance » du donné à l'explication (elle peut revêtir des formes très différentes). On sera alors amené à chercher une nouvelle description. Faut-il dire que celle-ci sera plus fidèle que la première ? Non, car elle l'aura 
reconstruite même si elle l'inclut. Par exemple, pour qu'une gaffe sociale soit interprétée comme un acte manqué, il faudra la relier à d'autres comportements du "même type ", qui auront eu lieu à d'autres moments et en toutes autres circonstances. De la sorte, la nouvelle description se révélera être une description différente; non seulement elle est plus étendue que la première mais elle en reformule aussi la teneur, plus ou moins globalement : c'est ainsi que l'on passera de la description « gaffe » à la description « acte manqué ».

51 Il n'y a pas une "question de la description », les débats portent uniquement sur son interprétation théorique pourvu qu'elle soit la même pour différents points de vue, c'està-dire pourvu que les descriptions rapportent le même état de choses. Si tel n'est pas le cas, cela ne signifie pas que l'on a «différentes descriptions d'un même fait » et que la bonne description est la description la plus proche de la réalité. On n'en saura jamais rien, c'est une illusion réaliste de prétendre qu'il peut y avoir, cognitivement, des faits « sousjacents ", antérieurs à leurs descriptions. Des descriptions qui ne coïncident pas décrivent des faits différents, la description que l'on estimera bonne est celle qui s'inscrit le mieux dans le cadre de nos attentes. Les théories ne décrivent pas la réalité, elles l'instituent cognitivement, la question de la description recouvre celle des attentes descriptives, qui est la seule bonne question - et c'est la théorie, non la description, qui s'avérera adéquate ou non à la réalité cognitive ainsi instituée. Les termes du conventionnalisme s'inversent, on ne choisit pas entre diverses descriptions d'un même fait mais entre des hypothèses qui orientent différemment les intentions descriptives, sur le fond de ce qu'il faut appeler les critères cognitifs de la réalité. Ils constituent des croyances qui ne sont pas les mêmes chez tous les observateurs, à l'exception de la croyance à la perception.

\section{NOTES}

1. R. Langacker, Foundation : of Cognitive Grammar, vol. II, Descriptive Application, Stanford, Stanford University Press, 1991, p. 295-298. On change l'exemple de Langacker qui propose F. a cassé le verre, proposition qui tend à présupposer un contexte, à savoir le comportement de Floyd ou un intérêt particulier porté sur le verre.

2. S'inspirant de Twardowski et de Husserl (II ${ }^{\mathrm{e}}$ Recherche Logique), S. Lesnievski a développé à partir de 1916 une logique nominaliste des parties et des touts. Elle se proposait d'éviter les antinomies de la théorie des ensembles, en considérant les relations élément/ensemble et sousensemble/ensemble comme des interprétations de la relation parties-touts. Les écrits principaux de Lesnievski sont Les fondements de la théorie générale des ensembles [1916, en polonais]; Sur les fondements de la mathématique [1927-1930, en polonais, trad. anglaise 1983] ; Esquisse d'un nouveau système des fondements de la mathématique [1929, en allemand].

3. Au sens, par exemple, de la théorie mertonienne de la «cumulation » des résultats successifs des enquêtes sociologiques. R. K. Merton, Social Theory and Social Structure, Glencoe, Free Press, 1957.

4. E. Husserl, Idées directrices pour une phénoménologie... [1913], trad. fr., Paris, Gallimard, 1963, § 67, p. 218 ; la traduction est ici remaniée. 
5. Ibid., §68, p.221. Cf. aussi Logique formelle et logique transcendantale [1929], Paris, Presses universitaires de France, 1957, 16 («Évidence de la clarté et évidence de la distinction») qui comprend trois sections : "Distinction et confusion », "Distinction et clarté », "Clarté de la possession des choses elles-mêmes et clarté de l'anticipation ».

6. Exemplarische Einzelheiten, dans la traduction "Cas individuels qui servent d'exemple ", Idées directrices..., op. cit., § 69, p. 222.

7. Ibid., $\$ 74$, p. 236, Husserl souligne «anexactes» et «concepts morphologiques », nous, «types ». Husserl ajoute que « le fait que » ces concepts ont « des sphères fluantes d'application » n'est pas « une tare qu'il faut leur imputer ».

8. Ibid., § 75, p. 240.

9. Ibid., § 69, p. 222.

10. R. Descartes, Méditations [ $1^{\text {re }}$ éd. en latin, 1641 ; trad. fr. revue par Descartes, 1647] : «Prenons pour exemple ce morceau de sucre, etc. ", Méd., II.

11. E. Kant, Métaphysique des Mœurs [1797], « Doctrine de la Vertu », § 52 et note. Je me permets de renvoyer à mon article « Exemple et pierre de touche chez Kant » [en portugais], Analise, 15, 1991. 12. Aussi, les controverses scientifiques - par essence, des situations d'incertitude cognitive «s'incarnent» souvent en des exemples. Pour en donner un Exempel avec quelque chose de Beispiel, la controverse publique entre Cuvier et Geoffroy Saint-Hilaire [1830] sur « l'unité du plan de composition zoologique » est vite passée de la discussion des méthodes aux « variations de l'os hyoïde » chez les vertébrés.

13. Respectivement dans Réponse à la question : qu'est-ce que les Lumières ? [1784], La religion dans les limites de la simple raison [1793], Le conflit des facultés [1798].

14. E. Kant, Critique de la raison pure [1781, $2^{\mathrm{e}}$ éd. 1787], B 172-174; cf. aussi la préface aux Fondements de la Métaphysique des Mours [1785].

15. E. Kant, La religion dans les limites de la simple raison, op. cit., Doctrine II, sect. I-B.

16. E. Husserl, Expérience et jugement [Erfahrung und Urteil, 1939], Paris, Presses universitaires de France, 1970, § 17.

17. Ibid., titre du § 19 .

18. "L'esthétique est descriptive. Ce qu'elle fait est d'attirer l'attention de quelqu'un sur certains traits, de placer les choses côte à côte afin de faire ressortir ces traits ", L. Wittgenstein, Les Cours de Cambridge (1932-1935), Mauvezin, Trans-Europ-Repress, 1992, p. 56 (souligné par Wittgenstein). Cf. aussi Investigations philosophiques [1953 pour la première édition anglaise], Paris, Gallimard, 1961, §570: «Les concepts nous mènent a des investigations. Ils sont l'expression de notre intérêt, et le dirigent ». Wittgenstein revient en d'autres endroits sur l'« intéressant ».

19. G. Lakoff, Women, Fire and Dangerous Things. What Categories Reveal about the Mind, Chicago, University of Chicago Press, 1987.

20. J. W. von Goethe, Übereinstimmung und Verschiedenheit, in Zur Morphologie, Hamburger Ausgabe, Hambourg, C. Wegner, vol. XIII, 1955, p. 63 ; Ähnlichkeit, Norm, allgemeiner Bild, Vergleichungskanon, Muster des Ganzen, in Erster Entwurf einer allgemeinen Einleitung in die vergleichende Anatomie, ausgehend von der Osteologie, ibid., p. 171-172.

21. Ibid., p. 173.

22. La sociologie récente des "sciences de laboratoire " a mis en évidence le caractère éidétique, comme l'écrit M. Lynch en se référant à Husserl, des documents, images ou "inscriptions » (diagrammes, courbes, etc.) qui sont les descriptions du physicien. Une typicité descriptive très riche y est à l'œuvre, cf. F. Gil, "De l'épistémologie à la philosophie, par le laboratoire ", in R. Guesnerie et F. Hartog, eds, Études sur les sciences et les techniques, Paris, Éd. de l'EHESS, 1999.

23. E. Mach, «Über Gedankenexperimente ", in Erkenntnis und Irrtum, $5^{\mathrm{e}}$ édition, Leipzig, J. A. Barth, 1926, p. 183 sq.

24. P. Duhem, La théorie physique, son objet et sa structure, Paris, Alcan, 1905, p. 331-332. 
25. J. Rawls, A Theory of Justice, Oxford, Oxford University Press, 1971, § 24, p. 136-138 (trad. fr., Paris, Seuil, 1987).

26. P. Strawson, Individuals [1959], Londres, Methuen, 1964, p.9. Strawson se propose de «dévoiler les structures les plus générales de notre structure conceptuelle» (ibid.), plus exactement la catégorialité de la pensée de l'individu saisie à ses sources: le sujet logicolinguistique (on pourrait évoquer aussi les « descriptions définies » de Russell), l'identification des particuliers, physiques (corps) ou phénoménaux (sons), les personnes enfin.

27. L. Wittgenstein, Investigations philosophiques, op. cit., § 90.

28. Ibid., § 291.

29. On adapte le cas imaginé par Ryle («The Thinking of Thoughts. What is Le penseur doing?», in Collected Papers, Londres, Hutchinson, t. II, Collected Essays 1929-1968, 1971), rediscuté par C. Geertz, «La description dense » (in The Interpretation of Cultures, New York, Basic Books, 1973), traduit dans ce même numéro, p. 73-105.

30. Ces points de vue abstraits sont également «typiques ", ainsi que les disciplines scientifiques. On a associé la typicité à l'objet de la description parce que c'est là qu'elle est « critique » et aussi pour mettre symétriquement en valeur la liberté de choix du point de vue théorique. Mais les points de vue se laissent également catégoriser.

31. La surdétermination du donné est autre chose que la compatibilité apparente des descriptions appartenant à différents univers du discours. La production d'un tic à fondement psychologique sera facilitée par une ligne de moindre résistance neurologique sans que cela implique pour autant que le tic constitue en même temps un réflexe nerveux.

\section{RÉSUMÉS}

Opération au fondement de l'objectivité, la description ne s'évalue pas pour autant à l'aune d'un critère réaliste. La description institue le fait qu'elle décrit, elle ne le reproduit pas. Les descriptions se font par rapport à des attentes explicatives, dans le cadre d'une hypothèse théorique plus ou moins thématisée. Mais elles ne sont pas arbitraires, le regard descriptif est guidé par les « saillances » de l'objet, dont tiennent compte les catégories de la description, tels le type et l'exemple. Sans être des copies, ces derniers ne sont pas non plus des interprétations. Cette étude s'attache à déterminer leur statut ainsi que la position épistémologique des descriptions dont le rôle consiste à fixer momentanément le fait pour conduire à une interrogation théorique.

Description is not evaluated according to realist criteria although it is an operation which establishes objectivity. It institutes but does not reproduce the fact described. Descriptions are made in relationship to expectations of explanations in the frame of a theoretical hypothesis. But they are not arbitrary. The descriptive gaze is guided by the striking features of the object which are taken in consideration by categories of description such as type and example. These latter are neither copies nor interpretations. This study seeks to determine the status as well as the epistemological position of descriptions whose role consists in momentarily establishing the fact in order to lead to a theoretical questioning. 


\section{AUTEUR}

FERNANDO GIL

Fernando Gil (EHESS), philosophe, s'intéresse aux problèmes de la preuve. Ses ouvrages les plus récents sont Traité de l'évidence (Grenoble, J. Millon, 1993), Viagens do Olhar (Porto, C. das Letras, 1998) et Modos da evidência (Lisbonne, INCM, 1998). 